\title{
Holographic storage and multiplexing in azopolyester blends using low energy pulses down to $2 \mathrm{~ms}$
}

\author{
C. Berges, ${ }^{1}$ I. Javakhishvili, ${ }^{2}$ S. Hvilsted, ${ }^{2}$ C. Sánchez-Somolinos, ${ }^{1}$ and R. Alcalá ${ }^{1, a)}$ \\ ${ }^{1}$ Departamento de Física de la Materia Condensada, Instituto de Ciencia de Materiales de Aragón (ICMA), \\ CSIC-Universidad de Zaragoza, C/Pedro Cerbuna 12, 50009 Zaragoza, Spain \\ ${ }^{2}$ Department of Chemical and Biochemical Engineering, Danish Polymer Centre, Technical University \\ of Denmark, Building 227, DK-2800 Kgs. Lyngby, Denmark
}

(Received 15 January 2013; accepted 1 May 2013; published online 14 May 2013)

\begin{abstract}
Three different blends containing side-chain azobenzene polyesters and poly(methyl methacrylate) homopolymers have been prepared for recording volume holographic polarization gratings using $488 \mathrm{~nm}$ light pulses. The final azo content in the blends has been decreased down to $0.2 \mathrm{wt}$. \%, and their morphologies have been investigated by transmission electron microscopy. Stable and rewritable polarization gratings have been recorded, and 20 gratings have been multiplexed using $2 \mathrm{~ms}$ pulses of low energy $\left(4 \mathrm{~mJ} / \mathrm{cm}^{2}\right)$. The equilibrium values of the diffraction efficiency were higher than $5 \times 10^{-5}$. C 2013 AIP Publishing LLC. [http://dx.doi.org/10.1063/1.4805076]
\end{abstract}

In recent decades, polymers containing azobenzene chromophores have been investigated as promising photocontrollable materials. ${ }^{1-4}$ Under irradiation with polarized light in the absorption region of the azo units (visible and UV), a preferential orientation of these units perpendicular to the polarization vector of the incident light can be induced due to the trans-cis-trans photoisomerizations of the azobenzenes. This generates optical anisotropy in polymers, which is appealing for several applications. One of these applications is holographic data storage that provides high values of data storage capacity and fast information transfer rates. ${ }^{5-9}$

Side-chain liquid crystalline (LC) azopolyester homopolymers have been widely investigated in the last years as promising holographic storage materials. The photoinduction of stable anisotropy and holographic polarization gratings has been reported. ${ }^{10}$ High values of birefringence (0.3) and dichroism (0.8) has been achieved under continuous light excitation. Besides, high efficiency diffraction gratings have been recorded in these materials. ${ }^{1-15}$

Most of the holographic studies in azopolymers have been performed on thin films (up to several microns) due to the high optical absorption of azobenzene containing polymers at the recording wavelength that limits light penetration through the film. However, a great increase in storage capacity can be obtained by means of volume holography that usually needs thicker films (up to hundreds of microns). The way to increase light penetration is to reduce azobenzene content. In this context, several groups have investigated holographic storage in films of block copolymers (BCs) as well as in blends of BCs and a homopolymer that does not absorb light at the recording wavelength. ${ }^{16-25}$

Thus, Häckel et al. ${ }^{22}$ have been able to store a stable holographic grating in $10 \mu \mathrm{m}$ thick films of a diBC containing azobenzene in one of the blocks. Due to microphase separation of the blocks, no surface relief gratings, that are deleterious for volume holographic storage, were created. The same group has studied the multiplexing of holographic gratings in thick films $(1.1 \mathrm{~mm})$ of blends containing an

${ }^{\text {a)} E l e c t r o n i c ~ m a i l: ~ r a l c a l a @ u n i z a r . e s ~}$ azobenzene block copolymer. Up to 20 holograms with grating efficiencies higher than $10^{-5}$ were multiplexed but using long writing times (of the order of seconds) and high energy density $\left(10 \mathrm{~J} / \mathrm{cm}^{2}\right) .^{23,24}$ Diffraction efficiencies above $10^{-5}$ and writing times in the ms range (or below) have been suggested as appropriate for holographic storage. ${ }^{26}$

In some previous papers, ${ }^{25,27}$ we have studied blends consisting of an azomethacrylic diBC and poly(methyl methacrylate) (PMMA) homopolymer containing $1 \mathrm{wt}$ \% azobenzene. It was demonstrated that up to 20 stable holographic polarization gratings were multiplexed in a $500 \mu \mathrm{m}$ thick film by irradiating with light pulses down to $10 \mathrm{~ms}$, achieving diffraction efficiencies higher than $10^{-5}$.

With the goal of improving holographic storage by reducing recording pulse duration and energy while keeping diffraction efficiency and multiplexing capability, we have extended our studies to other polymers containing azopolyester units. Thus, two BCs containing an azopolyester block and a PMMA non-absorbing block have been synthesized with the purpose of lowering the wt. \% azobenzene content in polyester compounds, while maintaining the azoazo molecular interactions. ${ }^{28}$ However, the achieved photoinduced birefringence and dichroism when irradiating with linearly polarized $488 \mathrm{~nm}$ light were unstable and lower than those corresponding to the azopolyester homopolymer in the two BCs. This was attributed to the lack of microstructure in one of the BCs and to the small size of the azobenzene nanospheres $(15-20 \mathrm{~nm})$ in the other one. Using blends of the latter BC with an azopolyester homopolymer, we have demonstrated that when adding a sufficiently high amount of the azopolyester, the photo-induced optical response of the $\mathrm{BC}$ was improved. However, a macrophase separation of azopolyester domains (size up to $500 \mathrm{~nm}$ ) was observed, together with the nanospheres corresponding to the $\mathrm{BC}$. Because of the big size of some of these domains, visible light scattering was observed. Besides, the azo content of these blends was too high to be used in volume holographic storage.

Trying to reduce the azobenzene content as well as the size of the azo domains, a ternary blend containing the $\mathrm{BC}$, the azopolyester homopolymer, and a PMMA homopolymer 


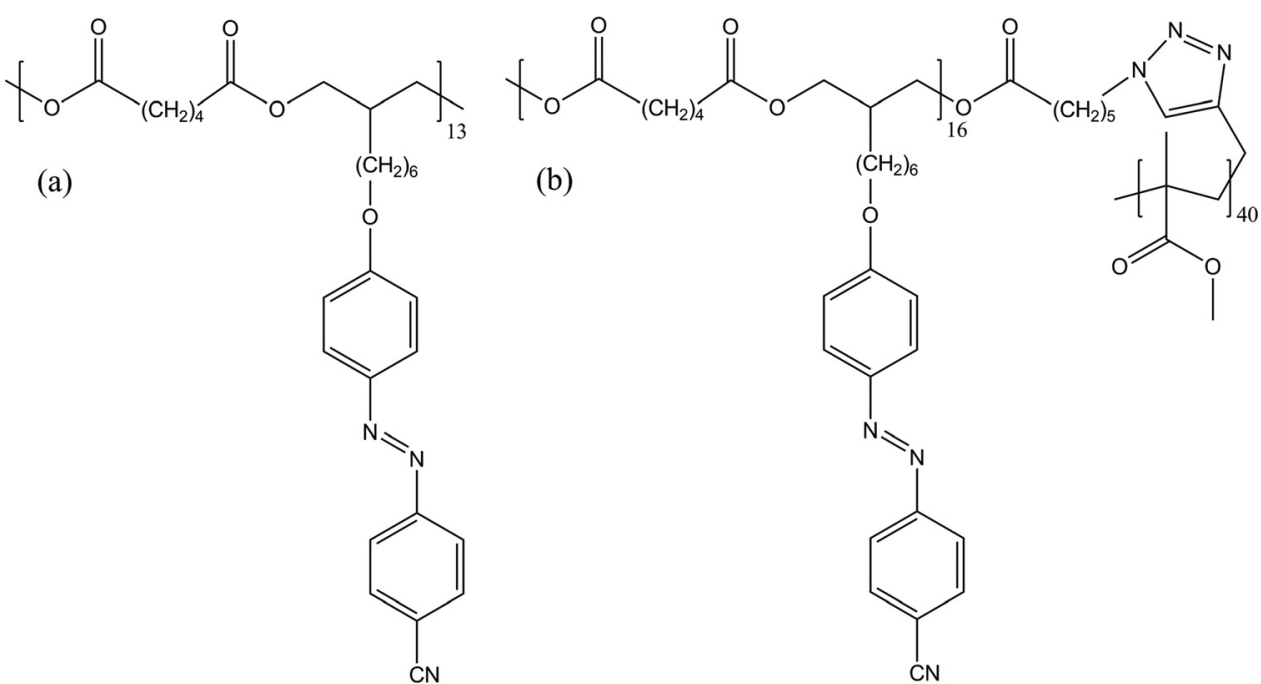

FIG. 1. Chemical structure of the azopolyester homopolymer (a) and BC (b) used for preparation of the studied blends. having a similar molecular weight $\left(\bar{M}_{n}=30000 \mathrm{~g} / \mathrm{mol}\right)$ as that of the PMMA forming the $\mathrm{BC}$, was prepared. The final azo content in this ternary blend was lowered down to 0.2 wt. \% (blend 1). Besides, two other binary blends (with the same azobenzene content) composed by the azopolyester homopolymer and PMMA homopolymers of $\bar{M}_{n}=30000 \mathrm{~g} /$ mol (blend 2) and $\bar{M}_{n}=90000 \mathrm{~g} / \mathrm{mol}$ (blend 3) were also prepared. In the present paper, we report on the recording and multiplexing of holographic polarization gratings in thick films of these blends using $488 \mathrm{~nm}$ light pulses down to $2 \mathrm{~ms}$ and low energy density $\left(4 \mathrm{~mJ} / \mathrm{cm}^{2}\right)$.

The BC containing a PMMA block and an azobenzene polyester block has been synthesized by "click" chemistry coupling of the preformed blocks, which in turn were obtained by Atom Transfer Radical Polymerization (ATRP) and polycondensation, respectively. The azopolyester homopolymer used for the preparation of blend 1 (see below) was also obtained by polycondensation $\left(\bar{M}_{n}=6600 \mathrm{~g} / \mathrm{mol}\right.$, Polydispersity Index (PDI): 1.85). The structures of the BC and the azopolyester homopolymer are shown in Fig. 1. Details of the synthesis and characterization of the $\mathrm{BC}$ and the azopolyester homopolymer are reported elsewhere. ${ }^{28} \mathrm{~A}$ PMMA homopolymer $\left(\bar{M}_{n}=30000 \mathrm{~g} / \mathrm{mol}\right)$ was also used to prepare blend 1 . This blend is composed of $0.38 \mathrm{wt} . \%$ of the BC, 0.16 wt. \% of the azopolyester homopolymer, and 99.46 wt. \% of the PMMA homopolymer. The azobenzene content of blend 1 is $0.2 \mathrm{wt}$ \%, with $0.04 \mathrm{wt} . \%$ of the total azo content coming from the $\mathrm{BC}$ and $0.16 \mathrm{wt} \%$ from the azobenzene polyester. Since most of the azo content of blend 1 comes from the added azopolyester homopolymer, other two blends ( 2 and 3 ) also containing 0.2 wt. \% azobenzene were prepared for comparison. These binary blends are obtained by simply mixing the azopolyester homopolymer with two PMMA homopolymers $\left(\bar{M}_{n}=30000 \mathrm{~g} / \mathrm{mol}\right.$ and $\left.\bar{M}_{n}=90000 \mathrm{~g} / \mathrm{mol}\right)$. All the blends were prepared in the same way by dissolving the corresponding amounts of the different components in dichloromethane, filtering the solution with Teflon filters $(0.2 \mu \mathrm{m}$ pore size), and evaporating the solvent at room temperature (RT).

Thick films (about $500 \mu \mathrm{m}$ ) were prepared by first pressing blend powder into pellets at $200{ }^{\circ} \mathrm{C}$ under vacuum (to eliminate air bubbles) and cooling it down to RT. A piece of the obtained pellet was then sandwiched at $200^{\circ} \mathrm{C}$ between two glass plates using spacers to get the desired thickness. The optical density (OD) of these thick films at $488 \mathrm{~nm}$ was about 0.25 for all the blends (measured with a Varian Cary 500 UV-visible-IR). This allows a good penetration of the $488 \mathrm{~nm}$ light through the complete film (0.56 of the incident light intensity goes through the film). Before performing holographic storage measurements, all the films were heated up to $80^{\circ} \mathrm{C}$ (above the isotropic phase transition temperature of the polyester) for $1 \mathrm{~min}$ and rapidly quenched by cooling to RT.

Holographic polarization gratings have been recorded in the $500 \mu \mathrm{m}$ thick films of the blends by irradiation with two oppositely circularly polarized $488 \mathrm{~nm}$ light beams from an $\mathrm{Ar}^{+}$laser creating the interference polarization pattern. The experimental set-up has been given elsewhere. ${ }^{16}$ Light pulses were obtained with a light shutter (Uniblitz model VMMT1) that can provide pulses with lengths down to $2 \mathrm{~ms}$. The angle between the two beams was about $6^{\circ}$ giving place to a diffraction grating period of about $2.5 \mu \mathrm{m}$. Diffraction efficiency of the recorded gratings was measured using a circularly polarized beam from a He-Ne laser at $633 \mathrm{~nm}$ incident at the Bragg angle and a Si photodetector to characterize the 0 th and +1 st orders. Multiplexing experiments were performed by rotating the film around an axis perpendicular to the incidence plane of the $488 \mathrm{~nm}$ light.

Samples for transmission electron microscopy (TEM) were obtained from the same blends as the films used for holographic measurements. Slices of about $100 \mathrm{~nm}$ thick were cut from those blends (using a Leica ultramicrotome EM UC6 equipped with a Diatome Ultra $35^{\circ}$ diamond knife) and picked up on carbon coated copper grids. The slices were exposed to $\mathrm{RuO}_{4}$ vapor that preferentially stains azobenzene aromatic rings. ${ }^{29}$ TEM was measured using a JEOL-2000 FXIII transmission electron microscope. In the micrographs, black regions correspond to the azobenzene domains stained with $\mathrm{RuO}_{4}$ and white regions correspond to the PMMA matrix.

As said above, a reduction of both light absorption and scattering with respect to those of the blends reported in Ref. 28 is required for volume holographic storage. This has been achieved by making blends 1,2 , and 3 with an 


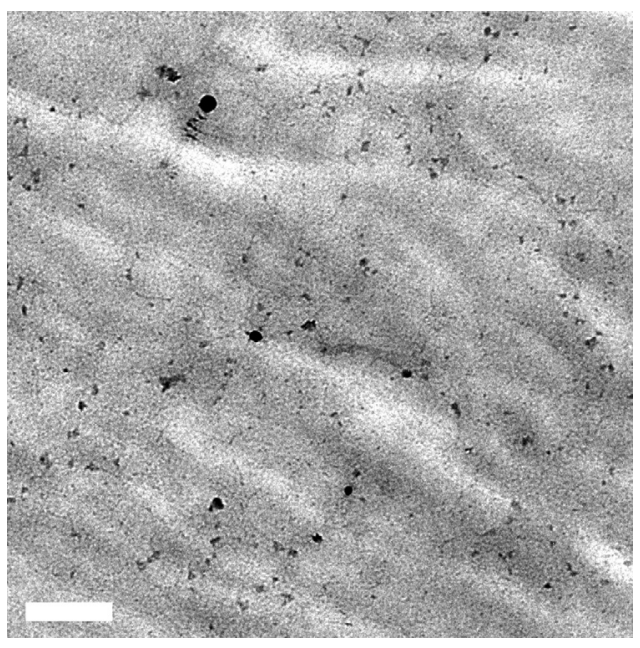

FIG. 2. TEM micrograph corresponding to blend 3. The size of the white bar is $500 \mathrm{~nm}$. Black dots correspond to azobenzene domains.

azobenzene content of $0.2 \mathrm{wt} \%$. The morphology of these blends was investigated by TEM. Similar microstructure was observed in the three blends. As an example, we give in Fig. 2 a micrograph corresponding to blend 3. A macrophase separation was still observed but the azobenzene domain size decreased (size up to $150 \mathrm{~nm}$ ). Because of this, visible light scattering became much lower and good optical quality films were obtained.

The recording of holographic polarization gratings in the Bragg regime has been investigated in thick films $(500 \mu \mathrm{m})$ of these blends. As for morphological studies, the results are similar for the three blends. From a practical point of view, preparation of blend 1 involves polymers that are more laborious to synthesize than simpler homopolymers employed in blends 2 and 3. Besides, due to the different $\bar{M}_{n}$ values of the PMMA homopolymers used in these last two blends, films prepared from blend 2 are more brittle than those of blend 3 . Because of this, we have focused our discussion on blend 3 .

It is known that the photoinduced response of LC azopolyester polymers is very sensitive to different storage conditions. In particular, thermal treatments as well as blue and red light illuminations can modify that response in a significant way. ${ }^{13-15,30-32}$ We have tried several conditions, and selected those that give high and stable values for the diffracted intensity of volume holographic gratings. After an initial annealing of the films at $80^{\circ} \mathrm{C}$ for $1 \mathrm{~min}$, they were irradiated for $5 \mathrm{~s}$ with a single circularly polarized $488 \mathrm{~nm}$ light beam $\left(2 \mathrm{~W} / \mathrm{cm}^{2}\right)$ to erase any previously stored information. It has been found that reproducible results are obtained after this irradiation.

In the first experiments, holographic gratings were recorded using a light pulse of $10 \mathrm{~ms}$ and an energy density of $20 \mathrm{~mJ} / \mathrm{cm}^{2}$ in each of the beams. The recording pulse was shot $5 \mathrm{~s}$ after the irradiation with the single $488 \mathrm{~nm}$ light beam was switched off. From the beginning of irradiation and all along the experiment films were illuminated with a circularly polarized He-Ne laser beam $(633 \mathrm{~nm}$ and power density of $1 \mathrm{~W} / \mathrm{cm}^{2}$ ) used to measure the diffraction efficiency of the photoinduced grating. Time evolution of this diffracted efficiency is given in Fig. 3. It shows a fast

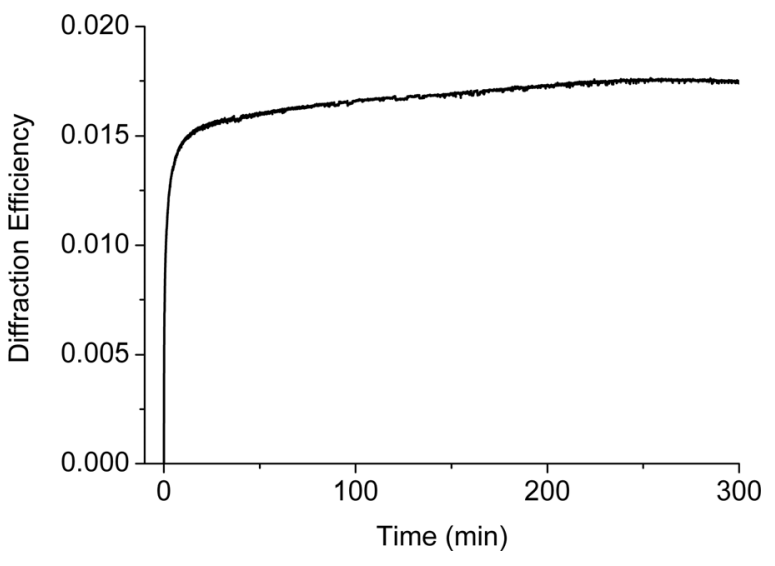

FIG. 3. Time evolution of the diffraction efficiency of a polarization grating stored in a thick film of blend $3(500 \mu \mathrm{m})$ with one $10 \mathrm{~ms}, 20 \mathrm{~mJ} / \mathrm{cm}^{2}$ pulse of $488 \mathrm{~nm}$ light.

increase to a value about $1.5 \times 10^{-2}$ in approximately $10 \mathrm{~min}$ after the recording pulse. Then it keeps growing slowly, saturates at about $1.7 \times 10^{-2}$ in $300 \mathrm{~min}$, and remains stable. This efficiency is two orders of magnitude higher than that previously found in a $1 \mathrm{wt}$ \% azobenzene blend containing an azomethacrylic BC and a PMMA homopolymer, under similar recording conditions. ${ }^{27}$

Holographic polarization gratings have also been recorded in blend 3 using a $2 \mathrm{~ms}$ pulse. The other irradiation conditions were the same as those used with the $10 \mathrm{~ms}$ pulse experiments. Time evolution of the diffraction efficiency during $300 \mathrm{~min}$ after the light pulse is similar to that of the $10 \mathrm{~ms}$ light pulse but the final efficiency is about $5 \times 10^{-3}$. On the other hand, angular multiplexing of up to 20 gratings has been investigated. In this case, (see Fig. 4) the reported results were achieved using $2 \mathrm{~ms}, 4 \mathrm{~mJ} / \mathrm{cm}^{2} 488 \mathrm{~nm}$ light pulses and a He-Ne laser power density of $100 \mathrm{~mW} / \mathrm{cm}^{2}$. The gratings recorded in these conditions were stable and the diffraction efficiency was similar for all of them and higher than $5 \times 10^{-5}$. Besides, those films were rewritable. Reproducible results were obtained after erasing the recorded gratings with a single circularly polarized $488 \mathrm{~nm}$ light beam.

Finally, we have studied the angular selectivity of the Bragg polarization grating after stabilization of the

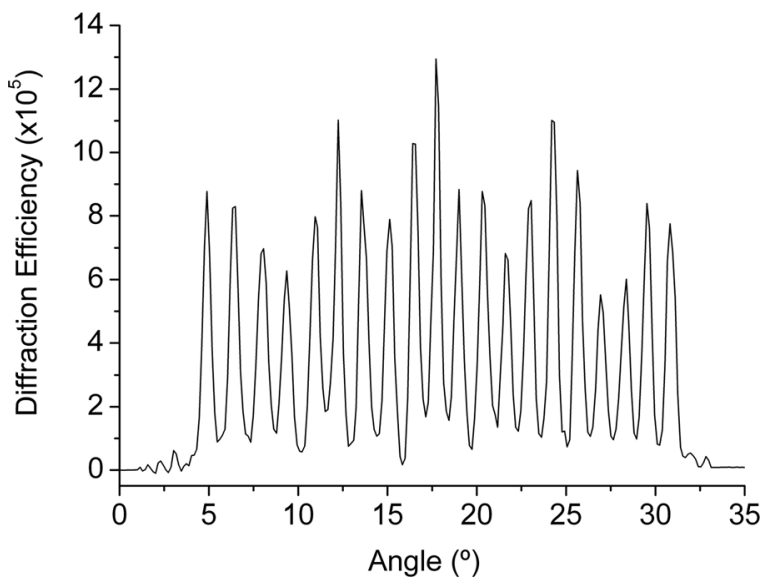

FIG. 4. Equilibrium values of diffraction efficiencies of 20 multiplexed gratings in a thick film of blend 3 using $488 \mathrm{~nm}$ light pulses $(2 \mathrm{~ms}$ and $4 \mathrm{~mJ} / \mathrm{cm}^{2}$ ). 


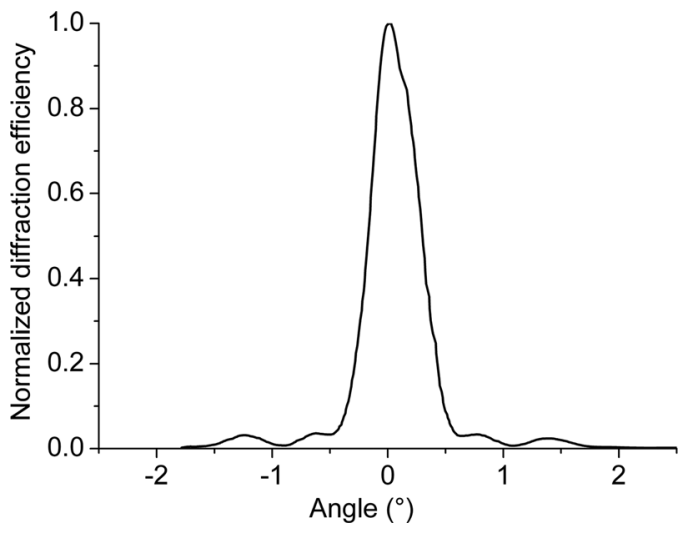

FIG. 5. Angular selectivity of a grating recorded in a $500 \mu \mathrm{m}$ thick film of blend 3 using $488 \mathrm{~nm}$ light pulses $\left(2 \mathrm{~ms}\right.$ and $\left.4 \mathrm{~mJ} / \mathrm{cm}^{2}\right)$.

diffraction efficiency induced with one $2 \mathrm{~ms}$ light pulse. The diffraction efficiency as a function of the rotation angle around an axis perpendicular to the incident plane of $488 \mathrm{~nm}$ light is shown in Fig. 5. As the Bragg angle was detuned, diffraction efficiency decreased to almost zero and then, successive side lobes of decreasing diffraction efficiency, characteristic of Bragg volume gratings, appeared. The angular selectivity was measured as the half width of the diffracted band, giving a value of $0.5^{\circ}$ for the two irradiation conditions.

In conclusion, the recording of volume holographic polarization gratings has been investigated in three azopolyester containing blends: A ternary blend consisting of an azopolyester-PMMA BC, an azopolyester homopolymer, and a PMMA homopolymer, and two binary blends consisting of the same azopolyester homopolymer and two PMMA homopolymers of different molecular weight. In the three blends, the final azobenzene content was reduced down to 0.2 wt. \%. Azobenzene domains with sizes smaller than $150 \mathrm{~nm}$ have been observed. With these blends, good optical quality thick films $(500 \mu \mathrm{m})$ have been prepared.

Volume holographic polarization gratings have been stored in these films using short and low energy $488 \mathrm{~nm}$ light pulses (down to $2 \mathrm{~ms}$ and $4 \mathrm{~mJ} / \mathrm{cm}^{2}$, respectively). Final stable diffraction efficiency of $5 \times 10^{-3}$ can be achieved with light pulses of $2 \mathrm{~ms}$. This efficiency is an order of magnitude higher than the best previously reported for azomethacrylic blends with higher azobenzene content $(1 \mathrm{wt} . \%)$ and using light pulses of $10 \mathrm{~ms}$ and higher energy density $\left(50 \mathrm{~mJ} / \mathrm{cm}^{2}\right)$.

Up to 20 holographic polarization gratings have been multiplexed in $500 \mu \mathrm{m}$ thick films of these blends by using $488 \mathrm{~nm}$ light pulses of $2 \mathrm{~ms}$ and $4 \mathrm{~mJ} / \mathrm{cm}^{2}$. Equilibrium values of the diffraction efficiencies higher than $5 \times 10^{-5}$ were achieved in all the gratings. These results show a clear improvement (shorter pulse length, lower energy, and higher diffraction efficiency) with respect to the results previously reported in the literature.
This work was supported by the Spanish MINECO Project No. MAT2011-27978-C02-02, Gobierno de Aragón, and FEDER funding (EU).

${ }^{1}$ Y. Zhao and T. Ikeda, Smart Light-Responsive Materials: AzobenzeneContaining Polymers and Liquid Crystals (Wiley-Interscience, 2009).

${ }^{2}$ H. Yu and T. Ikeda, Adv. Mater. 23, 2149 (2011).

${ }^{3}$ Z. Mahimwalla, K. G. Yager, J. Mamiya, A. Shishido, A. Priimagi, and C. J. Barret, Polym. Bull. 69, 967 (2012).

${ }^{4}$ A. Emoto, E. Uchida, and T. Fukuda, Polymers 4, 150 (2012).

${ }^{5}$ T. Bieringer, "Photoaddressable polymers," in Holographic Data Storage, edited by H. J. Coufal, D. Psaltis, and G. T. Sincerbox (Springer, New York, 2000), p. 209.

${ }^{6}$ A. Shishido, Polym. J. 42, 525 (2010).

${ }^{7}$ A. S. Matharu, S. Jeeva, and P. S. Ramanujam, Chem. Soc. Rev. 36, 1868 (2007).

${ }^{8}$ F. K. Bruder, R. Hagen, T. Rölle, M. S. Weiser, and T. Fäcke, Angew. Chem., Int. Ed. 50, 4552 (2011).

${ }^{9}$ S. Hvilsted, C. Sánchez, and R. Alcalá, J. Mater. Chem. 19, 6641 (2009).

${ }^{10}$ I. Zebger, M. Rutloh, U. Hoffmann, J. Stumpe, H. W. Siesler, and S. Hvilsted, J. Phys. Chem. A 106, 3454 (2002).

${ }^{11}$ P. S. Ramanujam, C. Holme, S. Hvilsted, M. Pedersen, F. Andruzzi, M. Paci, E. L. Tassi, P. Magagnini, U. Hoffman, I. Zebger, and H. W. Siesler, Polym. Adv. Technol. 7, 768 (1996).

${ }^{12}$ S. Hvilsted, F. Andruzzi, C. Kulinna, H. W. Siesler, and P. S. Ramanujam, Macromolecules 28, 2172 (1995).

${ }^{13}$ P. S. Ramanujam, S. Hvilsted, and F. Andruzzi, Appl. Phys. Lett. 62, 1041 (1993).

${ }^{14}$ C. Sánchez, R. Alcalá, S. Hvilsted, and P. S. Ramanujam, Appl. Phys. Lett. 77, 1440 (2000).

${ }^{15}$ C. Sánchez, R. Alcalá, S. Hvilsted, and P. S. Ramanujam, Appl. Phys. Lett. 78, 3944 (2001).

${ }^{16}$ P. Forcén, L. Oriol, C. Sánchez, F. J. Rodríguez, R. Alcalá, S. Hvilsted, and K. Jankova, Eur. Polym. J. 43, 3292 (2007).

${ }^{17}$ P. Forcén, L. Oriol, C. Sánchez, R. Alcalá, K. Jankova, and S. Hvilsted, J. Appl. Phys. 103, 123111 (2008).

${ }^{18}$ Y. Naka, H. Yu, A. Shishido, and T. Ikeda, Mol. Cryst. Liq. Cryst. 498, 118 (2009).

${ }^{19}$ H. Yu, Y. Naka, A. Shishido, and T. Ikeda, Macromolecules 41, 7959 (2008).

${ }^{20}$ H. Audorff, K. Kreger, R. Walker, D. Haarer, L. Kador, and H. W. Schmidt, Adv. Polym. Sci. 228, 59 (2010).

${ }^{21}$ Y. Zhao and J. He, Soft Matter 5, 2686 (2009).

${ }^{22}$ M. Häckel, L. Kador, D. Kropp, C. Frenz, and H. W. Schmidt, Adv. Funct. Mater. 15, 1722 (2005).

${ }^{23}$ M. Häckel, L. Kador, D. Kropp, and H. W. Schmidt, Adv. Mater. 19, 227 (2007).

${ }^{24}$ T. Breiner, K. Kreger, R. Hagen, M. Häckel, L. Kador, A. H. E. Müller, E. J. Kramer, and H. W. Schmidt, Macromolecules 40, 2100 (2007).

${ }^{25}$ P. Forcén, L. Oriol, C. Sánchez, F. J. Rodríguez, R. Alcalá, S. Hvilsted, and K. Jankova, Eur. Polym. J. 44, 72 (2008).

${ }^{26}$ J. Ashley, M. P. Bernal, G. W. Burr, H. Coufal, H. Guenther, J. A. Hoffnagle, C. M. Jefferson, B. Marcus, R. M. Macfarlane, R. M. Shelby, and G. T. Sincerbox, IBM J. Res. Dev. 44, 341 (2000).

${ }^{27}$ C. Berges, L. Oriol, M. Piñol, C. Sánchez, and R. Alcalá, Opt. Mater. 35, 1095 (2013).

${ }^{28}$ C. Berges, I. Javakhishvili, S. Hvilsted, C. Sánchez, and R. Alcalá, Macromol. Chem. Phys. 213, 2299 (2012).

${ }^{29}$ J. S. Trent, J. I. Scheinbeim, and P. R. Couchman, Macromolecules 16, 589 (1983).

${ }^{30}$ M. Kidowaki, T. Fujiwara, S. Morino, K. Ichimura, and J. Stumpe, Appl. Phys. Lett. 76, 1377 (2000).

${ }^{31}$ J. Minabe, K. Kawano, and Y. Nishikata, Appl. Opt. 41, 700 (2002).

${ }^{32}$ F. J. Rodríguez, C. Sánchez, B. Villacampa, R. Alcalá, R. Cases, M. V. Collados, S. Hvilsted, and M. Strange, Polymer 45, 6003 (2004). 